Recent Insights into the Physics of the Sun and Heliosphere:

Highlights from $\mathrm{SOHO}$ and Other Space Missions

IAU Symposium, Vol. 203, 2001

P. Brekke, B. Fleck, and J. B. Gurman eds.

\title{
Flare-CME Events Associated With a Superactive Region
}

\author{
J. Wang, J. Zhang, T. Wang, C. Zhang \\ National Astronomical Observatories, Chinese Academy of Sciences \\ Beijing 100012, China \\ Y. Liu \\ Hansen Experimental Physics Laboratory \\ Stanford University, USA \\ N. Nitta and G. L. Slater \\ Lockheed Martin Solar and Astrophysics Laboratory \\ Palo Alto, CA 94304, USA
}

\begin{abstract}
In a few recent studies, 8 coronal mass ejections (CMEs) were identified to associate with a superactive region, AR 8100 . They were all flare-CME events, It is found that the region is characterized by vigorous flux emergence and an imbalance of magnetic flux in two polarities. With the continuous flux emergence and its driven flux cancellation, the EUV dimming associated with flare activity increased in area. Whenever the dimming extended to an area of more than $50 \times 50$ square degrees on the solar surface, a flare in the region became CME-associated.
\end{abstract}

\section{Introduction}

Coronal mass ejections (CMEs) are the most spectacular form of solar magnetic activity. Great efforts and important progress have been made since the earliest detection of CMEs. However, systematic attempt has rarely been made to associate coronal mass ejections with disc sources so far (Howard et al. 1997).

In a few recent studies (Maia et al. 1999; Dermendjiev et al. 1999; Delannée and Aulanier, 2000; Delannée et al. 2000), 8 coronal mass ejections (CMEs) are identified to associate with a superactive region, AR 8100 . They were all associated with flares (Delannée et al. 2000). They took place in the period from November 3 to 6 when the region rotated from a position near central meridian to the west limb, thus enabled us to view CMEs from different aspects.

In this analysis we focus on the following questions: (1) what characterizes this CME-productive active region, (2) what are the key magnetic evolutions in the course of the flare-CME events, and (3) why a surface activity, e.g., a localized flare, becomes CME-associated. 


\section{Flare-CME Events Initiated in this Region}

In Table 1 we listed all the 8 flare-CME events which were clearly identified to initiate from this superactive region (see Delannée et al. 2000).

Table 1. Flare-CME Events

\begin{tabular}{lllccl}
\hline Number & Date & Flare Imp. & Flare $^{*}$ & Dimming $^{* *}$ & CME in C2 \\
\hline 1 & Nov.3 & C8.6/sb & $04: 32$ & $05: 03$ & $06: 10$ halo \\
2 & & M1.4/1b & $09: 03$ & $09: 34$ & $11: 11$ halo \\
3 & & M4.4/ & $10: 28$ & $10: 50$ & $11: 44$ halo \\
4 & Nov.4 & X2.1/2b & $05: 52$ & $06: 15$ & $06: 10$ halo \\
5 & Nov.5 & C7.0/1n & $06: 17$ & $07 ; 26$ & $07: 29$ \\
6 & & C6.8/ & $11: 30$ & $12: 01$ & $12: 10$ \\
7 & & /1f & $23: 25$ & $00: 15$ & $00: 18$ \\
8 & Nov.6 & X9.4/2b & $11: 49$ & $12: 15$ & $12: 10$ \\
\hline \hline
\end{tabular}

${ }^{*}$ - beginning; ${ }^{* *}$ - at maximum; ${ }^{* * *}$ - first appearance

Huairou Solar Observing Station observed the whole flare processes in $\mathrm{H} \alpha$ and $\mathrm{H} \beta$ lines of the first and 4-th events. A careful examination of SOHO EIT and LASCO/C2 movies has revealed some common properties of these events: 1) They initiated as flares first; 2) They had disk signature - an extended EUV dimming, covering an area larger than $50 \times 50$ square degrees on solar surface; 3 ) No classical 3 components - bright fronts, dark cavity, and filament core were identified.

\section{Characteristics of the Source Region}

There were, at least, 5 emerging flux regions (EFRs) in AR 8100, which were marked by numbers in Figure 1. EFR 2 was really of long-duration. Its flux emergence continued from Nov.1 to Nov.6. Seven of the 8 CMEs were initiated as the flares appearing on the boundary between EFR 2 and pre-existing negative flux. Delannée and Aulanier (2000) refer one of these flares as a 'bald patch flare'. The bald patch is just the site where EFR 2 was intruding into the nearby negative flux.

The CMEs did not happen at the early phase of flux emergence. The first CME associated with the region initiated when EFRs 1, 3, 4 and 5 were fully developed, and EFR 2 was in fast growing. By the continuous flux emergence and its driven flux cancellation, the magnetic fields in this region had established some global connectivity with other active and quiet regions, which were widely separated.

It is noticed that the dimming is not solely the disk signature of CMEs. From Nov.1 to early Nov.3. the dimming associated with flares was limited within the active region, the related flares were not CME-associated. However, in the later days the dimming extended from the region, crossed the equator, and reached the boundary of NOAA 8102 and other northern regions, covering an area of more than $50 \times 50$ square degrees on the Sun. Then, the relevant flares 


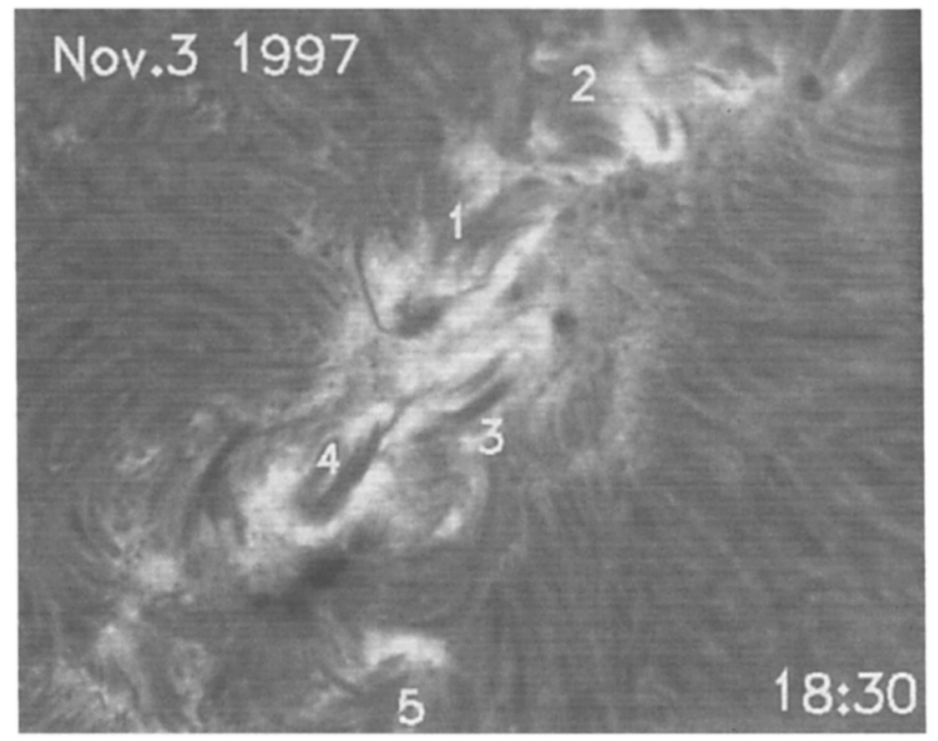

Figure 1. Big Bear $\mathrm{H} \alpha$ filtergram showing 5 EFRs which are marked by numbers. Arch filament systems for each EFR are clearly seen.

in the region became $\mathrm{CME}$-associated. Moreover, the bigger were the dimming areas, the more extended were the CMEs.

After removing the 180 degree ambiguity of field azimuth and projection effects in the vector magnetograms, we measured the total flux of the region. It reached $5.0 \times 10^{22} \mathrm{Mx}$ with net flux of $-0.5 \times 10^{22} \mathrm{Mx}$ when the first CME took place on Nov.3. This unbalanced flux indicated that a fraction of magnetic flux in the region was in the form of higher loops, connecting to widely separated regions on the Sun. We suggest that this is a characteristic of CME source regions.

Acknowledgments. J. Wang thanks Drs. J. Harvey, H. Hudson, K. Dere, B.C. Low, M. Pick, and T. Kosugi for valuable discussions. The work is supported by National National Science Foundation of China under Grants of 1979090 and 19973009.

\section{References}

Delannée, C. and Aulanier, G., 1999, Sol. Phys. 190, 107.

Delannée, C., Deleboudinière, J.-P. and Lamy, P., 2000 A\&A, 355,725.

Dermendjiev, V.N., Bochev, A.Z., Koleva, K.Zh., Kokotanekova, J.St., 1999, in Proc. 9-th European Meeting on Sol. Phys. (ESA SP-448), p971.

Howard, R.A., 1997, in N. Crooker et al.(eds) Coronal Mass Ejection, Geophysical Monograph 99, p.17.

Maia. D., Vourlidas, A., Pick, M., Howard, R., Schenn, R., and Magalhães, A., 1999, JGR, 104(A6), 12507. 